\title{
Health Disparities Among Sexual and Gender Minority Adults in Ohio
}

\author{
Hannah E. Albrechta ${ }^{1,2}$; Mira L. Katz; Megan E. Roberts ${ }^{1}$
}

${ }^{1}$ Division of Health Behavior and Health Promotion, College of Public Health, The Ohio State University, Columbus, OH

${ }^{2}$ The Fenway Institute, Fenway Health, Boston, MA

Corresponding Author: Megan E. Roberts, 1841 Neil Avenue, 353 Cunz Hall, Columbus, OH 43210, (614) 292-4647, roberts.1558@osu.edu

Submitted September 11, 2021 Accepted November 12, 2021 Published January 28,2022 https://doi.org/10.18061/ojph.v4i2.8561

\section{ABSTRACT}

Background: Many documented disparities associated with health behaviors and access to health care impact the health outcomes of sexual and gender minorities (SGM). The current study aimed to gain insight into the health disparities experienced by SGM adults living in Ohio to obtain data to plan future health-related programs.

Methods: A secondary data analysis was conducted using 2018 Ohio Behavior Risk Factor Surveillance System (BRFSS) data. Health behaviors, health care access, and health outcomes were analyzed by sexual orientation ( $n=11301$ ) and gender identity $(n=11426)$ to determine health disparities faced by sexual minority males, sexual minority females and transgender individuals.

Results: Significant SGM health disparities in Ohio are related to substance use (ie, binge drinking, current smoking status, e-cigarette use, and marijuana use); poor physical and mental health status; lack of health care coverage; and experiencing COPD, emphysema, or chronic bronchitis (all $p<0.001$ ). In addition, fewer sexual minority females reported being within recommended breast cancer screening guidelines than heterosexual females $(p<0.05)$.

Conclusion: Sexual and gender minority adults in Ohio report disparities associated with several health behaviors and access to health care. These findings may be due to factors at the patient level (eg, experiencing minority stress), provider level (eg, implicit bias), system level (eg, discrimination), and/or society level (eg, lack of legal protections). Study results will be used to plan health campaigns and programs targeted to SGM adults and providers to achieve health equity for the SGM population in Ohio.

Keywords: Sexual and gender minorities; Health status disparities; Health behavior; Substance-related disorders; BRFSS; Secondary analysis

\section{INTRODUCTION}

Sexual and gender minorities (SGM) in the United States (US) are disproportionately impacted by many disparities that can negatively impact health outcomes. Some health behaviors are wellestablished as being disproportionately high among SGM, such as smoking, heavy alcohol consumption, not attaining enough sleep, not exercising within recommended guidelines, and not using certain preventive health screenings. ${ }^{1-4}$ For example, sexual minority (SM) women are less likely to utilize cervical cancer screenings (eg, the Papanicolaou test). ${ }^{2-4}$ Sexual and gender minorities report they often avoid utilizing health care due to anticipated discrimination. ${ }^{5}$

There are also numerous adverse health outcomes that disproportionately impact SGM, such as having a normal body weight being less prevalent among SM women than heterosexual women.1,6 Disparities have also been documented for physical health symptoms (eg, pain, insomnia) and physical health conditions (eg, diabetes, asthma, hypertension, cancer, and stroke).6,7 In contrast, other studies found no significant differences in disease diagnoses including hypertension, diabetes, high cholesterol, or heart dis- 
ease. ${ }^{8,9}$ Sexual minorities are also disproportionately impacted by mental health disparities such as having a higher prevalence of a major depressive episode and generalized anxiety disorder compared to their heterosexual peers. ${ }^{10}$

Despite the growing body of evidence documenting SGM health disparities in the US, important gaps in public health professionals' knowledge persist. For example, marijuana and e-cigarette use is currently rising in popularity in the general population with limited information available about its use among SGMs. ${ }^{11,12}$ Public health professionals also need more information about SGM's health care coverage, their utilization of preventive health screenings, and their ability to access health care. These health-related issues and others may contribute to the disparities documented for various health outcomes.

Previous work investigated health disparities among SGMs in Ohio for physical health status, mental health status, smoking prevalence, and excessive drinking prevalence. ${ }^{13}$ The current study expands upon this previous work by investigating additional health disparities such as substance use, utilization of preventive health screenings, and various health outcomes. ${ }^{13}$ We focused our investigation on Ohio, as this state's SGM population is estimated to be $4.3 \%$ of the total state population, and the SGM population has a varied profile in terms of socioeconomic status, race/ethnicity, and urban/rural residence. ${ }^{14,15}$ In addition, Ohio ranks $25^{\text {th }}$ in the nation for public support of SGM rights and acceptance. ${ }^{14}$ Ohio's history of SGM discrimination is evident through lawsuits challenging Ohio's ban on same-sex marriage, which led to the 2015 US Supreme Court decision declaring state-level bans on same-sex marriage unconstitutional. ${ }^{14}$ As part of minority stress theory, stigma prejudice, and discrimination experienced by SGM creates a stressful environment that can partially explain many of the health disparities among this population. ${ }^{16}$ Currently, Ohio does not have nondiscrimination statutes that include sexual orientation or gender identity as protected individual characteristics. ${ }^{14}$ Thus, this study was conducted to document health disparities among adults in Ohio in order to have evidence to develop public health education campaigns and programs targeted to SGM adults and providers to achieve health equity for the SGM population in Ohio.

\section{METHODS}

\section{Data Source}

The current study used the 2018 Behavioral Risk Factor Surveillance System (BRFSS) to determine the health behaviors and health outcomes among SGMs living in Ohio. ${ }^{17}$ The BRFSS is an annual random-digit-dialing state-based telephone survey administered to noninstitutionalized adults aged 18 years or older by health departments in all 50 states, District of Columbia, Guam, and Puerto Rico in collaboration with the Centers for Disease Control and Prevention (CDC). ${ }^{18}$ In 2018, the combined response rate (landline and cellular) for individuals surveyed in Ohio was $51.7 \%$ $(\mathrm{N}=12763) \cdot{ }^{19}$ As part of the sexual orientation and gender identi- ty module, self-report data were collected from 11529 individuals on their sexual orientation, and 11518 indicated their gender identity. Individuals who responded "I do not know" or "refused" were excluded from the analysis resulting in analytic samples of 11301 who reported sexual orientation and 11426 who indicated their gender identity.

\section{Measures}

Sexual Orientation and Gender Identity

To collect sexual orientation data, participants were asked, "Which of the following best represents how you think of yourself?" (gay or lesbian, straight, bisexual, something else). Participants were also asked, "Do you consider yourself to be transgender?" If they reported "yes," they were then asked, "Do you consider yourself to be male-to-female, female-to-male, or gender nonconforming?" Due to the small numbers of responses in some categories, sexual orientation was collapsed into 4 categories (ie, straight male, SM male, straight female, and SM female), and gender identity was collapsed into 2 categories (ie, transgender and cisgender). The SM categories included individuals who identified as gay, gay/lesbian, bisexual, or something else. Transgender included individuals who indicated transgender (male-to-female or female-to-male) and gender nonconforming. Respondents' birth sex was collected from a single item with responses of male or female.

\section{Demographic Variables}

We used CDC-derived variables calculated to classify respondents for income, level of education completed, age, and race/ethnicity. ${ }^{20}$ The variable for income included 5 categories (eg, $<\$ 15000$, $\$ 15000$ to $<\$ 25000, \$ 25000$ to $<\$ 35000, \$ 35000$ to $<\$ 50000$ and $\$ 50000$ or more). Level of education completed included 4 categories (eg, did not graduate high school, graduated high school, attended college or technical school, and graduated from college or technical school). Age contained 6 levels (eg, 18-24 years, 25-34 years, 35-44 years, $45-54$ years, 55-64 years, and 65 years or older). Lastly, the variable for race/ethnicity contained 5 categories (eg, White non-Hispanic, Black non-Hispanic, other race non-Hispanic, multiracial non-Hispanic, and Hispanic).

\section{Health-Related Variables}

Substance use was assessed for 4 outcomes: binge drinking, smoking status, current e-cigarette use, and marijuana use. Binge drinking was defined as males having 5 or more drinks on 1 occasion and females having 4 or more drinks on 1 occasion in the past 30 days. We recoded smoking to include current smoker (smoked at least 100 cigarettes in their lifetime and now smoke every day or some days), former smoker (smoked at least 100 cigarettes in their lifetime and currently do not smoke), and individuals who reported never smoking. Current e-cigarette use was indicated as current use (using every day or some days) and no current use. Marijuana use was coded as any use in the past month (yes, no).

Health care access was examined among respondents aged 18 to 64 years with 2 variables. First, health care coverage was defined 
as having health insurance, prepaid plans such as health maintenance organization (HMOs), or government plans such as Medicare or Indian Health Services. ${ }^{21}$ Second, we examined whether participants reported a routine checkup within the past year (any time less than 12 months ago), within the past 5 years, or more than 5 years ago (including never).

Receiving a preventive health screening included having been tested for HIV, receiving 1 or more of the recommended colorectal cancer screenings within the recommended time interval among respondents aged 50 to 75 years, ${ }^{22}$ having a prostate cancer screening within the past 2 years asked of male respondents aged 40 years and older, receiving a Pap smear within the past 3 years asked of female respondents aged 21 to 65 years with an intact cervix, and receiving a mammogram within the past 2 years asked of female respondents aged 40 years and older.

A CDC-derived variable was used for body mass index (BMI) categorizing participants as underweight $(<18.5)$, normal weight $(18.5$ to $<25.0)$, overweight $(25.0$ to $<30.0)$, or obese $(\geq 30.0)$. Leisure time physical activity was a CDC-derived variable indicating physical activity in the past 30 days other than a regular job. CDCderived variables were also used for self-report health status, physical health status, and mental health status. For physical and mental health status, respondents reported if they had no days, 1 to 13 days, or 14 or more days when their physical or mental health was not good. Additional health outcomes were self- reported history of coronary heart disease (CHD) or myocardial infarction (MI); skin cancer; other types of cancer; chronic obstructive pulmonary disease (COPD), emphysema, or chronic bronchitis; and diabetes. Due to small cell sizes, individuals who reported having diabetes during pregnancy were excluded from the analysis.

\section{Statistical Analysis}

For substance use, health care access, preventive health screenings, and health outcomes, the proportion of participant responses were calculated by sexual orientation and by gender identity. Chisquare tests were conducted to analyze the association between all outcome variables by sexual orientation and by gender identity. We compared SM females to straight females, SM males to straight males, and transgender individuals to cisgender individuals. Analyses were conducted using SPSS Statistics software (SPSS Inc, version 27). Statistical significance was set at $\mathrm{p}<0.05$.

\section{RESULTS}

The analytic sample reported that $40.4 \%$ identified as a straight male, $1.6 \%$ as an SM male, $55.4 \%$ as a straight female, and $2.5 \%$ as an SM female. In addition, $99.4 \%$ identified as cisgender and $0.6 \%$ as transgender. The majority of respondents for all sexual orientations and gender identities were White non-Hispanic and were aged 55 years or older (Table 1).

Table1. Demographic Characteristics of Ohio Adults who Participated in the BRFSS in 2018

\begin{tabular}{|c|c|c|c|c|c|c|}
\hline \multirow[t]{2}{*}{ Demographic characteristics } & \multicolumn{3}{|c|}{ Sexual orientation } & \multicolumn{3}{|c|}{ Gender identity } \\
\hline & $\begin{array}{l}\text { Straight Male } \\
(\mathrm{n}=4571) \\
\mathrm{n}(\%)\end{array}$ & $\begin{array}{l}\text { SM Male }{ }^{a} \\
(n=183) \\
n(\%)\end{array}$ & $\begin{array}{l}\text { Straight Female } \\
(n=6265) \\
n(\%)\end{array}$ & $\begin{array}{l}\text { SM Female }{ }^{b} \\
(n=282) \\
n(\%)\end{array}$ & $\begin{array}{l}\text { Transgender } \\
(n=70) \\
n(\%)\end{array}$ & $\begin{array}{l}\text { Cisgender } \\
(n=11356) \\
n(\%)\end{array}$ \\
\hline \multicolumn{7}{|l|}{ Sex } \\
\hline Male & $4571(100)$ & $183(100)$ & 0 & 0 & $30(42.9)$ & $4750(41.8)$ \\
\hline Female & 0 & 0 & $6265(100)$ & $282(100)$ & $40(57.1)$ & $6606(58.2)$ \\
\hline \multicolumn{7}{|l|}{ Income level } \\
\hline Less than $\$ 15000$ & $300(7.4)$ & $21(12.7)$ & $612(11.5)$ & $38(16.7)$ & $8(14.0)$ & $979(10.0)$ \\
\hline$\$ 15000$ to $<\$ 25000$ & $643(15.8)$ & $41(24.8)$ & $1096(20.7)$ & $66(28.9)$ & $15(26.3)$ & $1861(19.0)$ \\
\hline$\$ 25000$ to $<\$ 35000$ & 455 (11.2) & $25(15.2)$ & $664(12.5)$ & $30(13.2)$ & $10(17.5)$ & $1184(12.1)$ \\
\hline$\$ 35000$ to $<\$ 50000$ & $639(15.7)$ & $18(10.9)$ & $732(13.8)$ & $29(12.8)$ & $7(12.3)$ & $1415(14.5)$ \\
\hline$\$ 50000$ or more & 2027 (49.9) & $60(36.4)$ & $2201(41.5)$ & $65(28.5)$ & $17(29.8)$ & $4350(44.4)$ \\
\hline \multicolumn{7}{|l|}{ Education level } \\
\hline Did not graduate high school & $289(6.3)$ & $15(8.2)$ & $399(6.4)$ & $22(7.8)$ & $7(10.0)$ & $735(6.5)$ \\
\hline Graduated high school & $1712(37.5)$ & $62(33.9)$ & $2188(35.0)$ & 107 (37.9) & $27(38.6)$ & 4101 (36.2) \\
\hline Attended college or technical school & $1202(26.3)$ & $45(24.6)$ & $1816(29.0)$ & $82(29.1)$ & $23(32.9)$ & $3148(27.8)$ \\
\hline $\begin{array}{l}\text { Graduated from college or technical } \\
\text { school }\end{array}$ & $1359(29.8)$ & $61(33.3)$ & $1852(29.6)$ & $71(25.2)$ & $13(18.6)$ & $3351(29.6)$ \\
\hline \multicolumn{7}{|l|}{ Age } \\
\hline $18-24$ years & $265(5.8)$ & $19(10.4)$ & $215(3.4)$ & $36(12.8)$ & $7(10.0)$ & $531(4.7)$ \\
\hline $25-34$ years & $434(9.5)$ & $24(13.1)$ & $453(7.2)$ & $45(16.0)$ & $8(11.4)$ & $950(8.4)$ \\
\hline $35-44$ years & $468(10.2)$ & $19(10.4)$ & $585(9.3)$ & $40(14.2)$ & $5(7.1)$ & $1112(9.8)$ \\
\hline $45-54$ years & $689(15.1)$ & $24(13.1)$ & $978(15.6)$ & $34(12.1)$ & $11(15.7)$ & $1718(15.1)$ \\
\hline $55-64$ years & $1113(24.3)$ & $41(22.4)$ & $1450(23.1)$ & $42(14.9)$ & $14(20.0)$ & $2650(23.3)$ \\
\hline 65 years or older & $1602(35.0)$ & $56(30.6)$ & $2584(41.2)$ & $85(30.1)$ & $25(35.7)$ & 4395 (38.7) \\
\hline \multicolumn{7}{|l|}{ Race/Ethnicity } \\
\hline White non-Hispanic & $4030(89.4)$ & $157(87.2)$ & $5562(89.7)$ & $223(80.2)$ & $62(88.6)$ & $10005(89.2)$ \\
\hline Black non-Hispanic & $248(5.5)$ & $11(6.1)$ & $394(6.4)$ & $17(6.1)$ & $3(4.3)$ & $675(6.0)$ \\
\hline Other non-Hispanic & $77(1.7)$ & $3(1.7)$ & $82(1.3)$ & $9(3.2)$ & $3(4.3)$ & $173(1.5)$ \\
\hline Multiracial non-Hispanic & $83(1.8)$ & $5(2.8)$ & $89(1.4)$ & $15(5.4)$ & 0 & $196(1.7)$ \\
\hline Hispanic & $69(1.5)$ & $4(2.2)$ & $75(1.2)$ & $14(5.0)$ & $2(2.9)$ & $164(1.5)$ \\
\hline
\end{tabular}

$(\mathrm{N}=11301$ who reported sexual orientation and $\mathrm{N}=11426$ who reported gender identity)

asexual minority (SM) male: identifying as gay, bisexual, or something else

bSexual minority (SM) female: identifying as gay/lesbian, bisexual, or something else 


\section{Substance Use}

Sexual minority males (18.2\%) and SM females (13.2\%) had larger proportions of individuals who engaged in binge drinking compared to their heterosexual counterparts; $\chi^{2}(3, N=11162)=$ 214.41, $\mathrm{p}<.001$ (Table 2). No association was found between binge drinking and gender identity. Sexual minority males (31.1\%) and SM females (25.4\%) had larger proportions of respondents who reported being current smokers compared to straight males (19.6\%) and straight females (17.7\%); $\chi^{2}(6, \mathrm{~N}=11231)=162.19, \mathrm{p}<.001$. No association was found between smoking status and gender identity. Sexual minority females (10.0\%) and SM males (6.6\%) had a greater proportion that currently used e-cigarettes compared to their straight peers; $\chi^{2}(3, N=11291)=52.02, p<.001$. Sexual minority males $(17.1 \%)$ and SM females $(11.7 \%)$ had a significantly greater proportion of individuals who used marijuana in the past month compared to straight males (7.9\%) and straight females (3.8\%); $\chi^{2}(3, N=11253)=140.49, \mathrm{p}<.001$. Due to small cell sizes, a chisquare value could not be calculated to determine the association between current e-cigarette use and gender identity, and marijuana use and gender identity.

\section{Health Care Access}

Sexual minority males (11.1\%) and SM females (9.7\%) had the largest proportion of respondents who did not have health care coverage compared to straight males (8.2\%) and straight females $(5.6 \%) ; \chi^{2}(3, \mathrm{~N}=6898)=23.31, \mathrm{p}<.001$ (Table 3). Due to small cell sizes, a chi-square value could not be calculated to determine the association between health care coverage and gender identity. Straight females (87\%) and SM females (85.6\%) had a greater proportion of respondents who received a routine checkup within the past year compared to straight males (79.9\%) and SM males $(80.4 \%) ; \chi^{2}(6, N=11211)=114.53, p<.001$. No association was found between time since last routine checkup and gender identity.

\section{Preventive Health Screening}

A significant association was found between HIV testing and sexual orientation; $\chi^{2}(3, N=10936)=127.48, p<.001$ (Table 4). Sexual minority males (55.8\%) had the largest proportions of individuals who had ever been tested for HIV compared to SM females (43.4\%), straight males (25.4\%), and straight females (25.4\%). No association was found between HIV testing and gender identity. Out of respondents aged 50 to 75 years, a significantly larger proportion of straight females (71.1\%) and SM females $(72.7 \%)$ received the recommended colorectal cancer tests compared to straight males (68.0\%) and SM males (65.5\%); $\chi^{2}(3, N=6042)=7.80, p=.05$. No association was found between the following: colorectal cancer screening and gender identity, prostate cancer screening and sexual orientation, and prostate cancer screening and gender identity.

Additionally, no statistically significant relationship was found between cervical cancer screening and sexual orientation. Due to small cell sizes, a chi-square value could not be calculated to determine the association between cervical cancer screening and gender identity. There was a significant disparity with a larger proportion of straight females (74.8\%) aged 40 years or older receiving mammograms within the past 2 years compared to SM females $(67.0 \%) ; \chi^{2}(1, N=5390)=5.53, p=.019$. An even wider gap emerged when analyzing the association between breast cancer screening and gender identity; $\chi^{2}(1, N=5477)=4.40$, $\mathrm{p}=$.036. A larger proportion of cisgender females (74.6\%) aged

Table 2. Association of Substance Use Behaviors by Sexual Orientation and by Gender Identity Among Ohio Adults who Participated in the BRFSS in 2018

\begin{tabular}{|c|c|c|c|c|c|c|c|c|}
\hline \multirow[t]{2}{*}{ Behaviors } & \multicolumn{5}{|c|}{ Sexual orientation } & \multicolumn{3}{|c|}{ Gender identity } \\
\hline & $\begin{array}{l}\text { Straight Male } \\
(\mathrm{n}=4571) \\
\mathrm{n}(\%)\end{array}$ & $\begin{array}{l}\text { SM Male } \\
(n=183) \\
n(\%)\end{array}$ & $\begin{array}{l}\text { Straight Female } \\
(\mathrm{n}=6265) \\
\mathrm{n}(\%)\end{array}$ & $\begin{array}{l}\text { SM Female } \\
(\mathrm{n}=282) \\
\mathrm{n}(\%)\end{array}$ & $x^{2}$ & $\begin{array}{l}\text { Transgender } \\
(\mathrm{n}=70) \\
\mathrm{n}(\%)\end{array}$ & $\begin{array}{l}\text { Cisgender } \\
(\mathrm{n}=11356) \\
\mathrm{n}(\%)\end{array}$ & $\chi^{2}$ \\
\hline \multicolumn{5}{|l|}{ Binge drinking $^{a}$} & \multicolumn{3}{|l|}{$214.41^{\star \star \star}$} & 0.20 \\
\hline Yes & 762 (16.9) & 33 (18.2) & 487 (7.9) & $37(13.2)$ & & $9(13.4)$ & $1312(11.7)$ & \\
\hline No & $3737(83.1)$ & $148(81.8)$ & $5715(92.1)$ & $243(86.8)$ & & $58(86.6)$ & $9906(88.3)$ & \\
\hline \multicolumn{5}{|l|}{ Smoking status } & \multicolumn{3}{|l|}{$162.19^{\star * *}$} & 1.47 \\
\hline Current smoker & $892(19.6)$ & $56(31.1)$ & $1102(17.7)$ & $71(25.4)$ & & $17(24.3)$ & 2109 (18.7) & \\
\hline Former smoker & $1501(33.0)$ & $54(30.0)$ & $1518(24.4)$ & $77(27.6)$ & & $19(27.1)$ & $3164(28.0)$ & \\
\hline Never smoked & $2150(47.3)$ & $70(38.9)$ & 3609 (57.9) & $131(47.0)$ & & $34(48.6)$ & $6010(53.3)$ & \\
\hline \multicolumn{5}{|c|}{ Current e-cigarette use } & \multicolumn{3}{|l|}{$52.02^{\star * *}$} & - \\
\hline Yes & $190(4.2)$ & $12(6.6)$ & $178(2.8)$ & $28(10.0)$ & & $6(8.6)$ & $405(3.6)$ & \\
\hline No & $4374(95.8)$ & $171(93.4)$ & $6086(97.2)$ & $252(90.0)$ & & $64(91.4)$ & $10939(96.4)$ & \\
\hline \multicolumn{5}{|c|}{ Marijuana use in past month } & \multicolumn{3}{|l|}{$140.49 * * *$} & - \\
\hline Yes & $357(7.9)$ & $31(17.1)$ & $237(3.8)$ & $33(11.7)$ & & $11(15.9)$ & $650(5.8)$ & \\
\hline No & $4190(92.1)$ & $150(82.9)$ & 6007 (96.2) & $248(88.3)$ & & $58(84.1)$ & 10654 (94.2) & \\
\hline
\end{tabular}

$(\mathrm{N}=11301$ who reported sexual orientation and $\mathrm{N}=11426$ who reported gender identity)

$*=\mathrm{p} \leq .05$

${ }^{* *}=\mathrm{p}<.01$

$* * *=p<.001$

a Use in the past month

${ }^{b}$ Sexual minority (SM) male: identifying as gay, bisexual or something else

c Sexual minority (SM) female: identifying as gay/lesbian, bisexual or something else

-A chi-square value could not be calculated due to low cell size 
Table 3. Association of Health Care Access by Sexual Orientation and by Gender Identity Among Ohio Adults who Participated in the BRFSS in 2018

\begin{tabular}{|c|c|c|c|c|c|c|c|c|}
\hline \multirow[t]{2}{*}{ Health care access } & \multicolumn{5}{|c|}{ Sexual orientation } & \multicolumn{3}{|c|}{ Gender identity } \\
\hline & $\begin{array}{l}\text { Straight Male } \\
(\mathrm{n}=4571) \\
\mathrm{n}(\%)\end{array}$ & $\begin{array}{l}\text { SM Male }^{a} \\
(n=183) \\
n(\%)\end{array}$ & $\begin{array}{l}\text { Straight Female } \\
(n=6265) \\
n(\%)\end{array}$ & $\begin{array}{l}\text { SM Female }{ }^{b} \\
(n=282) \\
n(\%)\end{array}$ & $\chi^{2}$ & $\begin{array}{l}\text { Transgender } \\
(\mathrm{n}=70) \\
\mathrm{n}(\%)\end{array}$ & $\begin{array}{l}\text { Cisgender } \\
(n=11356) \\
n(\%)\end{array}$ & $\chi^{2}$ \\
\hline Health care coverage & & & & & $23.31^{\star \star *}$ & & & - \\
\hline Yes & $2683(91.8)$ & 112 (88.9) & $3447(94.4)$ & 177 (90.3) & & 40 (90.9) & $6403(93.1)$ & \\
\hline No & $241(8.2)$ & $14(11.1)$ & $205(5.6)$ & $19(9.7)$ & & $4(9.1)$ & 478 (6.9) & \\
\hline Routine checkup & & & & & $114.53^{\star * \star}$ & & & 3.20 \\
\hline Within past year & 3618 (79.9) & $144(80.4)$ & $5419(87.0)$ & $238(85.6)$ & & $56(80.0)$ & $9468(84.1)$ & \\
\hline Within past 5 years & $580(12.8)$ & $20(11.2)$ & $578(9.3)$ & $28(10.1)$ & & $7(10.0)$ & $1207(10.7)$ & \\
\hline $\begin{array}{l}5 \text { or more years } \\
\text { ago, or never }\end{array}$ & $329(7.3)$ & $15(8.4)$ & $230(3.7)$ & $12(4.3)$ & & $7(10.0)$ & $588(5.2)$ & \\
\hline
\end{tabular}

( $N=11301$ who reported sexual orientation and $N=11426$ who reported gender identity)

$*=p \leq .05$

$* *=p<.01$

$* * *=p<.001$

${ }^{a}$ Sexual minority (SM) male: identifying as gay, bisexual, or something else

${ }^{b}$ Sexual minority (SM) female: identifying as gay/lesbian, bisexual, or something else

-A chi-square value could not be calculated due to low cell size.

Table 4. Association of Preventive Health Screening Behaviors by Sexual Orientation and by Gender Identity Among Ohio Adults Who Participated in the BRFSS in 2018

\begin{tabular}{|c|c|c|c|c|c|c|c|c|}
\hline \multirow[t]{2}{*}{ Behaviors } & \multicolumn{5}{|c|}{ Sexual Orientation } & \multicolumn{3}{|c|}{ Gender Identity } \\
\hline & $\begin{array}{l}\text { Straight Male } \\
(\mathrm{n}=4571) \\
\mathrm{n}(\%)\end{array}$ & $\begin{array}{l}\text { SM Male }^{d} \\
(n=183) \\
n(\%)\end{array}$ & $\begin{array}{l}\text { Straight Female } \\
(n=6265) \\
n(\%)\end{array}$ & $\begin{array}{l}\text { SM Female } \\
(n=282) \\
n(\%)\end{array}$ & $\chi^{2}$ & $\begin{array}{l}\text { Transgender } \\
(\mathrm{n}=70) \\
\mathrm{n}(\%)\end{array}$ & $\begin{array}{l}\text { Cisgender } \\
(\mathrm{n}=11356) \\
\mathrm{n}(\%)\end{array}$ & $\chi^{2}$ \\
\hline \multicolumn{5}{|l|}{ HIV testing } & $127.48^{* \star *}$ & & & 1.35 \\
\hline Yes & $1121(25.4)$ & $101(55.8)$ & $1537(25.4)$ & $119(43.4)$ & & $22(32.4)$ & $2870(26.1)$ & \\
\hline No & $3301(74.6)$ & $80(44.2)$ & $4522(74.6)$ & $155(56.6)$ & & $46(67.6)$ & $8112(73.9)$ & \\
\hline \multicolumn{5}{|c|}{ Colorectal cancer screening } & $7.80^{*}$ & & & 0.42 \\
\hline Yes $^{a}$ & $1682(68.0)$ & $55(65.5)$ & $2409(71.1)$ & $72(72.7)$ & & $22(64.7)$ & $4228(69.8)$ & \\
\hline $\mathrm{No}^{\mathrm{a}}$ & $791(32.0)$ & $29(34.5)$ & $977(28.9)$ & $27(27.3)$ & & $12(35.3)$ & $1826(30.2)$ & \\
\hline \multicolumn{5}{|c|}{ Prostate cancer screening in past 2 years ${ }^{b}$} & 1.05 & & & 0.43 \\
\hline Yes & $1343(39.1)$ & $55(43.7)$ & $\ldots$ & $\ldots$ & & $5(31.3)$ & $1400(39.3)$ & \\
\hline No & $2091(60.9)$ & $71(56.3)$ & $\ldots$ & $\ldots$ & & $11(68.8)$ & $2161(60.7)$ & \\
\hline \multicolumn{5}{|c|}{ Cervical cancer screening in past 3 years ${ }^{c}$} & 3.23 & & & - \\
\hline Yes & $\ldots$ & $\ldots$ & $2235(80.7)$ & $109(74.7)$ & & $11(64.7)$ & $2339(80.4)$ & \\
\hline No & $\ldots$ & $\ldots$ & $534(19.3)$ & $37(25.3)$ & & $6(35.3)$ & $571(19.6)$ & \\
\hline \multicolumn{5}{|c|}{ Breast cancer screening in past 2 years ${ }^{b}$} & $5.53^{\star}$ & & & $4.40^{*}$ \\
\hline Yes & $\ldots$ & $\ldots$ & $3899(74.8)$ & $120(67.0)$ & & $20(58.8)$ & $4058(74.6)$ & \\
\hline No & $\ldots$ & $\ldots$ & $1312(25.2)$ & $59(33.0)$ & & $14(41.2)$ & $1385(25.4)$ & \\
\hline
\end{tabular}

( $N=11301$ who reported sexual orientation and $N=11426$ who reported gender identity)

$*=\mathrm{p} \leq .05$

$* *=p<.01$

$* * *=p<.001$

ages: 50 to 75 years

${ }^{b}$ Ages: 40 years and older

${ }^{c}$ Ages: 21 to 65 years

${ }^{\mathrm{d} S}$ Sexual minority (SM) male: identifying as gay, bisexual, or something else

eSexual minority (SM) female: identifying as gay/lesbian, bisexual, or something else

-A chi-square value could not be calculated due to low cell size.

...Not Applicable 
40 years or older received a mammogram within the past 2 years compared to transgender individuals (58.8\%).

\section{Health Outcomes}

Sexual minority males (30.2\%) and SM females (28.5\%) had an increased proportion of respondents who indicated fair or poor health status compared to straight males (21.8\%) and straight females $(21.0 \%) ; \chi^{2}(3, N=11275)=17.37, \mathrm{p}=.001$ (Table 5). A significant association was observed between physical health status and sexual orientation; $\chi^{2}(6, N=11123)=79.10, p<.001$. Sexual minority males (22.3\%) and SM females (22.4\%) had the largest proportion of individuals who indicated they experienced 14 days or more of poor physical health. Sexual minority males (22.3\%) and SM females (26.8\%) had greater proportions of individuals who experienced 14 or more days of poor mental health compared to straight males (10.5\%) and straight females (14.7\%); $\chi^{2}(6, N=11150)=271.08, p<.001$. No association was found between the following: health status and gender identity, physical health status and gender identity, and mental health status and gender identity.

A significant association was demonstrated between BMI and sexual orientation; $\chi^{2}(9, N=10722)=180.50, p<.001$. Sexual minority females $(40.3 \%)$ had the largest proportion and SM males (27.6\%) had the smallest proportion of individuals considered to be obese (BMI $\geq 30.0$ ). No association was found between BMI and gender identity; $\chi^{2}(3, N=10828)=1.46, p=.693$. Straight females (30.9\%) and SM females (34.8\%) had larger proportions of respondents who did not engage in leisure time physical activity outside of work in the past 30 days compared to their male counterparts; $\chi^{2}(3, \mathrm{~N}=11282)=31.27, \mathrm{p}<.001$. No association was found between leisure time physical activity and gender identity.

A significant association was found between skin cancer and sexual orientation; $\chi^{2}(3, N=11281)=9.68, p=.022$. Sexual minority males $(12.0 \%)$ had the largest proportion who had skin cancer in their lifetime, while SM females (4.6\%) had the lowest percentage. No association was found between skin cancer and gender identity. A significant relationship was also demonstrated between other types of cancer and sexual orientation; $\chi^{2}(3, N=11276)=31.24$, $\mathrm{p}<.001$. Straight females $(11.7 \%)$ had the highest percentage of individuals who had other types of cancer besides skin cancer in their lifetime, and straight males (8.4\%) had the lowest. Sexual minority males (10.9\%) and SM females (10.0\%) had similar proportions. No significant association was observed between other types of cancer and gender identity.

Straight males (14.4\%) and SM males (15.9\%) had a larger proportion of individuals who had a CHD or MI compared to their female counterparts; $\chi^{2}(3, N=11205)=75.22, p<.001$. No association was found between CHD or MI and gender identity. Sexual minority males (15.8\%) and SM females (16.4\%) had larger proportions of individuals who have had COPD, emphysema, or chronic bronchitis compared to straight males (10.3\%) and straight females $(12.9 \%) ; \chi^{2}(3, N=11267)=24.02, p<.001$. No association was found between COPD, emphysema, or chronic bronchitis and gender identity. No significant association was found between diabetes and sexual orientation, and between diabetes and gender identity.

\section{DISCUSSION}

The findings from the current study indicate the many health disparities experienced by the SGM population in Ohio. Consistent with previous research, 1,7,8,10,13,23 significant disparities were found for physical and mental health status, and substance use (ie, binge drinking, current smoking status, and marijuana use) among SM males and SM females. Additionally, a significant disparity emerged for e-cigarette use among SM females and SM males. Aligning with previous research,1,6,8 the current study also found SM females to have the largest proportion of individuals to be classified as obese. Contrasting previous research, ${ }^{1}$ the current study found SM females to have a significantly greater proportion of individuals who reported no leisure time physical activity in the past 30 days compared to their heterosexual peers. The lack of partaking in leisure time physical activity could partially explain the greater proportion of SM females classified as obese.

Novel contributions to the literature include the finding that there were disparities for experiencing COPD, emphysema, or chronic bronchitis among SM males and SM females. As tobacco smoking is the most common cause of COPD, the greater proportion of SM females and SM males who are current smokers could help explain the disparity for experiencing COPD, emphysema, or chronic bronchitis. ${ }^{24}$ Supporting previous research, ${ }^{8,9}$ the current study found limited differences between SGM and their heterosexual peers in reporting cardiovascular disease diagnoses like $\mathrm{CDH}$ or MI and diabetes, but found SMs were more likely to report cardiovascular risk factors such as substance use, increased BMI, and poor mental health. The significant difference found in reporting CDH or MI was between all males and females.

A significant disparity was demonstrated for breast cancer screening with a larger proportion of straight females reported receiving a mammogram within the past 2 years compared to SM females. Another new finding was an even wider disparity for gender identity with a significantly larger proportion of cisgender females receiving a mammogram in the past 2 years than transgender individuals. One possible explanation for this disparity is that transgender individuals may avoid seeking routine health care due to anticipated discrimination. ${ }^{5} \mathrm{~A}$ critical finding is the disparity that emerged for health care coverage among SM males and SM females. The disparity in health care coverage can limit access to care, which could partially explain the disparity in preventive health screenings.

\section{Limitations}

Telephone surveys were used to collect data for the 2018 BRFSS data set. It is important to note that telephone surveys exclude 


\begin{tabular}{|c|c|c|c|c|c|c|c|c|}
\hline \multirow[t]{2}{*}{ Health outcomes } & \multicolumn{5}{|c|}{ Sexual orientation } & \multicolumn{3}{|c|}{ Gender identity } \\
\hline & $\begin{array}{l}\text { Straight Male } \\
(\mathrm{n}=4571) \\
\mathrm{n}(\%)\end{array}$ & $\begin{array}{l}\text { SM Male } \\
(n=183) \\
n(\%)\end{array}$ & $\begin{array}{l}\text { Straight Female } \\
(n=6265) \\
n(\%)\end{array}$ & $\begin{array}{l}\text { SM Female }{ }^{d} \\
(n=282) \\
n(\%)\end{array}$ & $\chi^{2}$ & $\begin{array}{l}\text { Transgender } \\
(\mathrm{n}=70) \\
\mathrm{n}(\%)\end{array}$ & $\begin{array}{l}\text { Cisgender } \\
(n=11356) \\
n(\%)\end{array}$ & $\chi^{2}$ \\
\hline Health status & & & & & $17.37^{* *}$ & & & 0.05 \\
\hline $\begin{array}{l}\text { Excellent, very good, or } \\
\text { good }\end{array}$ & $3566(78.2)$ & $127(69.8)$ & $4943(79.0)$ & $201(71.5)$ & & $54(77.1)$ & $8866(78.3)$ & \\
\hline Fair or poor & 992 (21.8) & $55(30.2)$ & $1311(21.0)$ & $80(28.5)$ & & $16(22.9)$ & 2462 (21.7) & \\
\hline Physical health status & & & & & $79.10^{\star * \star}$ & & & 0.40 \\
\hline $\begin{array}{l}\text { No days physical health } \\
\text { was not good }\end{array}$ & $2887(64.1)$ & $99(55.3)$ & $3621(58.8)$ & $117(42.2)$ & & $38(56.7)$ & $6752(60.5)$ & \\
\hline $\begin{array}{l}1-13 \text { days physical health } \\
\text { was not good }\end{array}$ & $939(20.8)$ & $40(22.3)$ & $1543(25.0)$ & $98(35.4)$ & & $17(25.4)$ & $2624(23.5)$ & \\
\hline $\begin{array}{l}14 \text { days or more physical } \\
\text { health was not good }\end{array}$ & $680(15.1)$ & $40(22.3)$ & $997(16.2)$ & $62(22.4)$ & & $12(17.9)$ & $1793(16.1)$ & \\
\hline Mental health status & & & & & $271.08^{\star \star *}$ & & & 5.22 \\
\hline $\begin{array}{l}\text { No days mental health was } \\
\text { not good }\end{array}$ & $3325(73.8)$ & $98(54.7)$ & $3796(61.4)$ & $119(42.5)$ & & $43(63.2)$ & $7388(65.9)$ & \\
\hline $\begin{array}{l}1-13 \text { days mental health } \\
\text { was not good }\end{array}$ & $711(15.8)$ & $41(22.9)$ & $1482(24.0)$ & $86(30.7)$ & & $10(14.7)$ & $2331(20.8)$ & \\
\hline $\begin{array}{l}14 \text { days or more mental } \\
\text { health was not good }\end{array}$ & $471(10.5)$ & $40(22.3)$ & $906(14.7)$ & $75(26.8)$ & & $15(22.1)$ & $1485(13.3)$ & \\
\hline $\mathrm{BMI}$ & & & & & $180.50 * * *$ & & & 1.46 \\
\hline Underweight $(<18.5)$ & $45(1.0)$ & $3(1.7)$ & $115(2.0)$ & $7(2.7)$ & & 0 & $173(1.6)$ & \\
\hline Normal weight (18.5 to <25.0) & $953(21.2)$ & $49(27.1)$ & $1702(29.5)$ & $95(36.1)$ & & $15(23.4)$ & $2822(26.2)$ & \\
\hline Overweight $(25.0$ to <30.0) & $1849(41.1)$ & $79(43.6)$ & $1836(31.8)$ & $55(20.9)$ & & $25(39.1)$ & $3821(35.5)$ & \\
\hline Obese $(\geq 30.0)$ & $1655(36.8)$ & $50(27.6)$ & $2123(36.8)$ & $106(40.3)$ & & $24(37.5)$ & $3948(36.7)$ & \\
\hline Leisure time physical activity in & past 30 days & & & & $31.27^{* * \star}$ & & & 0.17 \\
\hline Yes & $3360(73.7)$ & $132(72.1)$ & $4321(69.1)$ & $184(65.2)$ & & $8027(70.8)$ & $48(68.6)$ & \\
\hline No & $1202(26.3)$ & $51(27.9)$ & $1934(30.9)$ & $98(34.8)$ & & $3310(29.2)$ & $22(31.4)$ & \\
\hline Skin cancer & & & & & $9.68^{*}$ & & & 0.57 \\
\hline Yes & $455(10.0)$ & $22(12.0)$ & $607(9.7)$ & $13(4.6)$ & & $5(7.1)$ & $1114(9.8)$ & \\
\hline No & $4105(90.0)$ & $161(88.0)$ & $5651(90.3)$ & $267(95.4)$ & & $65(92.9)$ & $10223(90.2)$ & \\
\hline Other types of cancer & & & & & $31.24^{* * *}$ & & & 1.67 \\
\hline Yes & $384(8.4)$ & $20(10.9)$ & $733(11.7)$ & $28(10.0)$ & & $4(5.7)$ & $1184(10.5)$ & \\
\hline No & 4177 (91.6) & $163(89.1)$ & $5518(88.3)$ & $253(90.0)$ & & $66(94.3)$ & $10145(89.5)$ & \\
\hline $\mathrm{CHD}$ or $\mathrm{Ml}^{\mathrm{a}}$ & & & & & $75.22^{\star \star}$ & & & 0.58 \\
\hline Yes & $654(14.4)$ & $29(15.9)$ & $577(9.3)$ & $23(8.3)$ & & $6(8.6)$ & $1294(11.5)$ & \\
\hline No & $3874(85.6)$ & $153(84.1)$ & $5641(90.7)$ & $254(91.7)$ & & $64(91.4)$ & $9966(88.5)$ & \\
\hline COPD, emphysema, or chronic & ronchitis ${ }^{b}$ & & & & $24.02^{* * *}$ & & & 0.36 \\
\hline Yes & $471(10.3)$ & $29(15.8)$ & $803(12.9)$ & $46(16.4)$ & & $10(14.3)$ & $1354(12.0)$ & \\
\hline No & $4085(89.7)$ & $154(84.2)$ & $5445(87.1)$ & $234(83.6)$ & & $60(85.7)$ & $9967(88.0)$ & \\
\hline Diabetes & & & & & 0.53 & & & 0.82 \\
\hline Yes & $788(17.3)$ & $31(16.9)$ & $1018(16.5)$ & $41(15.0)$ & & $10(14.5)$ & $1908(17.0)$ & \\
\hline No & $3680(80.6)$ & $146(79.8)$ & $5038(81.7)$ & $228(83.2)$ & & $58(84.1)$ & $9118(81.1)$ & \\
\hline Prediabetes or borderline & $96(2.1)$ & $6(3.3)$ & $110(1.8)$ & 5 (1.8) & & 1 (1.4) & 217 (1.9) & \\
\hline
\end{tabular}

( $N=11301$ who reported sexual orientation and $\mathrm{N}=11426$ who reported gender identity)

$*=p \leq .05$

$* *=p<.01$

$* * *=p<.001$

${ }^{a}$ Coronary heart disease (CHD) or myocardial infarction (MI)

${ }^{\mathrm{b}}$ Chronic obstructive pulmonary disease (COPD)

'Sexual minority (SM) male: identifying as gay, bisexual, or something else

${ }^{\mathrm{d}}$ Sexual minority (SM) female: identifying as gay/lesbian, bisexual, or something else 
individuals who do not have access to phones and individuals who have prepaid phones. Another limitation is that sexual orientation and gender identity variables are only part of the optional BRFSS modules, which hinders the ability of researchers to fully understand SGM health disparities across different regions.

Condensing the SM and transgender variables allowed for greater cell sizes and power needed for statistical analyses, but it limited the ability to analyze differences within sexual and gender identities. Notably, there were only 70 transgender individuals in the sample, so some analyses may have been underpowered to detect significant effects. A limitation of the current study is the lack of ability to analyze the interactions between SGM individuals and other intersecting identities, such as race, due to small sample sizes.

\section{PUBLIC HEALTH IMPLICATIONS}

The most critical SGM health disparities in Ohio are related to greater substance use (ie, binge drinking, current smoking status, e-cigarette use, and marijuana use); poor physical and mental health status; experiencing COPD, emphysema, or chronic bronchitis; lack of health care coverage; and reduced rates of receiving breast cancer screenings. These SGM disparities are likely due to several factors, including experiencing minority stress, anticipated discrimination, and a lack of legal protections. ${ }^{5,16,25-29}$ Public health action is critically needed to better understand and address these disparities. Education campaigns and programs targeted to SGM to address health issues such as substance use, mental health, and preventive screenings are vital. Additionally, programs aimed toward reducing bias among health care providers in Ohio may reduce anticipated discrimination among SGM and thereby increase their utilization of health care and preventive screenings. Establishing legislation that outlaws discrimination toward SGM in Ohio could also reduce the health disparities found by lessening the stigma, prejudice, and discrimination faced by this population. The Ohio Fairness Act is an example of legislation that seeks to clarify in Ohio that it is illegal to discriminate based on one's sexual orientation or gender identity, but it has yet to pass into law. ${ }^{30}$ Legal protections establishing the equal rights of SGM individuals and outlawing discrimination are critical to create lasting change in reducing SGM disparities.

\section{REFERENCES}

1. Cunningham TJ, Xu F, Town M. Prevalence of Five Health-Related Behaviors for Chronic Disease Prevention Among Sexual and Gender Minority Adults - 25 U.S. States and Guam, 2016. MMWR Morb Mortal Wkly Rep. 2018;67(32):888-893. https://doi.org/10.15585/mmwr.mm6732a4

2. Reiter PL, McRee A. Cervical cancer screening (Pap testing) behaviours and acceptability of human papillomavirus self-testing among lesbian and bisexual women aged 21-26 years in the USA.J Fam Plann Reprod Health Care. 2015;41:259-264. https://doi.org/10.1136/jfprhc-2014-101004

3. Agénor M, Krieger N, Austin SB, Haneuse S, Gottlieb BR. Sexual orientation disparities in Papanicolaou test use among US women: the role of sexual and reproductive health services. Am J Public Health. 2014;104

(2):e68-e73.

https://doi.org/10.2105/AJPH.2013.301548

4. Lee M, Jenkins WD, Adjei Boakye E. Cancer screening utilization by residence and sexual orientation. Cancer Causes Control. 2020;31 (10):951-964.

https://doi.org/10.1007/s10552-020-01339-4

5. Casey LS, Reisner SL, Findling MG, et al. Discrimination in the United States: experiences of lesbian, gay, bisexual, transgender, and queer Americans. Health Serv Res. 2019;54 Suppl 2(Suppl 2):1454-1466. https://doi.org/10.1111/1475-6773.13229

6. Trinh MH, Agénor M, Austin SB, Jackson CL. Health and healthcare disparities among U.S. women and men at the intersection of sexual orientation and race/ethnicity: a nationally representative crosssectional study. BMC Public Health. 2017;17:964. https://doi.org/10.1186/s12889-017-4937-9

7. Bränström R, Hatzenbuehler ML, Pachankis JE. Sexual orientation disparities in physical health: age and gender effects in a populationbased study. Soc Psychiatry Psychiatr Epidemiol. 2016;51(2):289-301. https://doi.org/10.1007/s00127-015-1116-0

8. Conron KJ, Mimiaga MJ, Landers SJ. A population-based study of sexual orientation identity and gender differences in adult health. Am J Public Health. 2010;100(10):1953 -1960. https://doi.org/10.2105/AJPH.2009.174169

9. Caceres BA, Brody A, Luscombe RE, et al. A systematic review of cardiovascular disease in sexual minorities. Am J Public Health. 2017;107

(4):e13-e21.

https://doi.org/10.2105/AJPH.2016.303630

10. Rice CE, Vasilenko SA, Fish JN, Lanza ST. Sexual minority health disparities: an examination of age-related trends across adulthood in a national cross-sectional sample. Ann Epidemiol. 2019;31:20-25. https://doi.org/10.1016/j.annepidem.2019.01.001

11. Uddin SMI, Osei AD, Obisesan OH, et al. Prevalence, trends, and distribution of nicotine and marijuana use in e-cigarettes among US adults: the Behavioral Risk Factor Surveillance System 2016-2018. Prev Med. 2020;139:106175.

https://doi.org/10.1016/j.ypmed.2020.106175

12. Bandi P, Cahn Z, Goding Sauer A, et al. Trends in e-cigarette use by age group and combustible cigarette smoking histories, U.S. adults, 20142018. Am J Prev Med. 2021;60(2):151-158. https://doi.org/10.1016/j.amepre.2020.07.026

13. Ohio Department of Health. 2019 Online State Health Assessment. Ohio Department of Health. Published 2019. Accessed September 2020. https://odh.ohio.gov/wps/portal/gov/odh/explore-data-and-stats/ interactive-applications/2019-Online-State-Health-Assessment

14. Mallory C, Brown TNT, Sears B. The Impact of Stigma and Discrimination Against LGBT People in Ohio. Published October, 2019. Accessed May 26, 2021.

https://williamsinstitute.law.ucla.edu/wp-content/uploads/ImpactLGBT-Discrimination-OH-Nov-2019.pdf

15. The Williams Institute. LGBT Demographic Data Interactive. LGBT Data \& Demographics. Published January, 2019. Accessed May 26, 2021. https://williamsinstitute.law.ucla.edu/visualization/lgbt-stats/? topic $=$ LGBT \&area $=39 \#$ density

16. Meyer IH. Prejudice, social stress, and mental health in lesbian, gay, and bisexual populations: conceptual issues and research evidence. 
Psycho Bull. 2003;129(5):674-697.

https://doi.org/10.1037/0033-2909.129.5.674

17. Centers for Disease Control and Prevention. 2018 BRFSS Data. United States: Centers for Disease Control and Prevention. Published 2019.

Accessed September, 2020.

https://www.cdc.gov/brfss/annual_data/annual_2018.html

18. Centers for Disease Control and Prevention. The Behavioral Risk Factor Surveillance System (BRFSS) Overview 2018. CDC. Published July 26, 2019. Accessed September, 2020.

https://www.cdc.gov/brfss/annual_data/2018/pdf/overview-2018508.pdf

19. Centers for Disease Control and Prevention. Behavioral Risk Factor Surveillance System 2018 Summary Data Quality Report. CDC. Published July 17, 2019. Accessed September, 2020.

https://www.cdc.gov/brfss/annual_data/2018/pdf/2018-sdqr508.pdf

20. Centers for Disease Control and Prevention. Calculated Variables in the 2018 Data File of the Behavioral Risk Factor Surveillance System (BRFSS). CDC. Updated May 24, 2019. Accessed September, 2020. https://www.cdc.gov/brfss/annual_data/2018/pdf/2018-calculatedvariables-version4-508.pdf

21. Centers for Disease Control and Prevention. LLCP 2018 Codebook Report. CDC. Published November 21, 2019. Accessed September, 2020.

https://www.cdc.gov/brfss/annual_data/2018/pdf/codebook18_llcpv2-508.pdf

22. U.S. Preventive Services Task Force. Final Recommendation Statement Colorectal Cancer: Screening. U.S. Preventive Services Task Force. Updated 2016. Accessed March 9, 2021.

https://www.uspreventiveservicestaskforce.org/uspstf/recommendation/ colorectal-cancer-screening\#fullrecommendationstart

23. Schuler MS, Prince DM, Breslau J, Collins RL. Substance use disparities at the intersection of sexual identity and race/ethnicity: results from the 2015-2018 National Survey on Drug Use and Health. LGBT Health. 2020;7(6):283-291.

https://doi.org/10.1089/lgbt.2019.0352

24. Mayo Clinic. Overview. COPD. Published April 15, 2020. Accessed March 9, 2021.

https://www.mayoclinic.org/diseases-conditions/copd/symptomscauses/syc-20353679

25. Frost DM, Lehavot K, Meyer IH. Minority stress and physical health among sexual minority individuals. J Behav Med. 2015;38(1):1-8. https://doi.org/10.1007/s10865-013-9523-8

26. Gonzales G, Ehrenfeld JM. The association between state policy environments and self-rated health disparities for sexual minorities in the United States. Int J Environ Res Public Health. 2018;15(6):1136. https://doi.org/10.3390/ijerph15061136

27. Lee JH, Gamarel KE, Bryant KJ, Zaller ND, Operario D. Discrimination, mental health, and substance use disorders among sexual minority populations. LGBT Health. 2016;3(4):258-265. https://doi.org/10.1089/lgbt.2015.0135

28. Valentine SE, Shipherd JC: A systematic review of social stress and mental health among transgender and gender non-conforming people in the United States. Clin Psychol Rev. 2018;66:24-38.

https://doi.org/10.1016/j.cpr.2018.03.003
29. Raifman J, Moscoe E, Austin SB, Hatzenbuehler ML, Galea S. Association of state laws permitting denial of services to same-sex couples with mental distress in sexual minority adults: a difference-in-difference-indifferences analysis. JAMA Psychiatry. 2018;75(7):671-677. https://doi.org/10.1001/jamapsychiatry.2018.0757

30. Equality Ohio. Ohio Fairness Act. Legislation. Accessed May 26, 2021 https://equalityohio.org/legislation/ofa-senate/ 